Johannes W.G. Janssen • Issei Imoto • Jun Inoue

Yutaka Shimada • Masakazu Ueda • Masayuki Imamura

Claus R. Bartram • Johji Inazawa

\title{
MYEOV, a gene at 11q13, is coamplified with CCND1, but epigenetically inactivated in a subset of esophageal squamous cell carcinomas
}

Received: April 26, 2002 / Accepted: May 17, 2002

\begin{abstract}
DNA amplifications at $11 \mathrm{q} 13$ are frequently observed in esophageal squamous cell carcinoma (ESC) and correlate with a malignant phenotype. Although this amplicon spans a region of several megabases and harbors numerous genes, CCND1 and EMS1 are thought to be the relevant candidates in ESC. We investigated whether the putative transforming gene MYEOV, mapping $360 \mathrm{~kb}$ centromeric to $C C N D 1$ and activated concomitantly with CCND1 in a subset of $\mathrm{t}(11 ; 14)(\mathrm{q} 13 ; \mathrm{q} 32)$ positive multiple myeloma cell lines, represents a target of 11q13 amplification in ESC. To evaluate the role of MYEOV in ESC, we tested 31 ESC cell lines and 48 primary tumors for copy number levels of $M Y E O V$ and demonstrated that MYEOV was always coamplified with $C C N D 1$. However, MYEOV expression levels correlated only inconsistently with DNA amplification data. Treatment with the demethylating agent 5-aza-2'-deoxycytidine restored MYEOV expression in a subset of cell lines exhibiting DNA amplification without high $M Y E O V$ expression, suggesting that MYEOV is transcriptionally silenced by a DNA methylation mechanism in most of the latter cell lines. Our results indicate that
\end{abstract}

J.W.G. Janssen · C.R. Bartram

Institute of Human Genetics, Ruprecht-Karls-University Heidelberg, Heidelberg, Germany

I. Imoto $\cdot \mathrm{J}$. Inoue $\cdot \mathrm{J}$. Inazawa $(\bowtie)$

Department of Molecular Cytogenetics, Medical Research Institute, Tokyo Medical and Dental University, 1-5-45 Yushima, Bunkyo-ku,

Tokyo 113-8510, Japan

Tel. +81-3-5803-5820; Fax +81-3-5803-0244

e-mail: johinaz.cgen@mri.tmd.ac.jp

J. Inoue

Theranostics Research Center (TRC), Otsuka Pharmaceutical Co. Ltd., Tokushima, Japan

Y. Shimada $\cdot$ M. Imamura

Department of Surgery, Surgically Basic Medicine, Kyoto University Graduate School of Medicine, Kyoto, Japan

M. Ueda

Department of Surgery, School of Medicine, Keio University, Tokyo, Japan

The first two authors contributed equally to this work.
MYEOV is a coamplified gene with CCND1 at 11q13, but its activation is sometimes inhibited by an epigenetic mechanism.

Key words 11q13 Amplification - Esophageal squamous cell carcinoma $\cdot M Y E O V \cdot C C N D 1 \cdot E M S 1 \cdot$ Epigenetic

\section{Introduction}

It has been reported that amplification at $11 \mathrm{q} 13$ is involved in esophageal squamous cell carcinoma (ESC) as well as in several other types of solid tumors (Yoshida et al. 1993; Schuuring 1995; Schwab 1998). In addition, there is evidence that this amplification is associated with malignant phenotypes of ESC, such as invasiveness, metastasis, and poor prognosis (Yoshida et al. 1993; Adelaide et al. 1995; Shinozaki et al. 1996). Within the 11q13 amplicon, CCND1 and EMS1 are the only genes known to be both amplified and overexpressed, thereby making them target genes in ESC as well as other tumors exhibiting the 11q13 amplification (Schuuring 1995; Hui et al. 1997). However, this amplicon spans a 3-5 $\mathrm{Mb}$ region, and comprises numerous genes that have not been well characterized thus far (Map Viewer, http://www.ncbi.nlm.nih.gov/cgi-bin/Entretz/ map-search).

The chromosomal band 11q13 is frequently involved in chromosomal translocations in various hematological malignancies (Mitelman et al. 1997), such as the translocation $\mathrm{t}(11 ; 14)(\mathrm{q} 13 ; \mathrm{q} 32)$ in a subset of B-cell malignancies, especially in non-Hodgkin lymphomas and multiple myeloma (MM) (Mitelman et al. 1997; Ronchetti et al. 1999; Chesi et al. 2000). This type of recurrent chromosomal alteration juxtaposes $C C N D 1$ to the $\operatorname{IgH}-5^{\prime} \mathrm{E} \mu$ enhancer at $14 \mathrm{q} 34.2$, resulting in the deregulation of $C C N D 1$ expression, which is considered to be a critical molecular event in the pathogenesis of $\mathrm{t}(11 ; 14)$ hematological malignancies (Nakamura et al. 1997). Through application of the NIH/3T3 tumorigenicity assay, MYEOV was recently identified as a novel transforming gene and mapped at 11q13 (Janssen et al. 
1999, 2000). Fine mapping of $M Y E O V$ by fluorescence in situ hybridization to extended DNA fibers showed that this gene was sublocalized $360 \mathrm{~kb}$ centromeric to $C C N D 1$. Interestingly, the breakpoints of MM cell lines with $\mathrm{t}(11 ; 14)$ were localized within this 360-kb region between MYEOV and $C C N D 1$. Furthermore, in a subset of MM cell lines with $\mathrm{t}(11 ; 14)$ and $C C N D 1$ overexpression, overexpression of $M Y E O V$ was revealed as well, suggesting that $M Y E O V$ might act as a second oncogene or as a modifier of B-cell transformation (Janssen et al. 2000).

All these findings prompted us to examine the role of $M Y E O V$ in esophageal carcinogenesis. We therefore investigated the amplification and expression status of MYEOV together with $C C N D 1$ and $E M S 1$ in our series of ESC cell lines as well as in primary tumors. Our results show $M Y E O V$ coamplification with $C C N D 1$ in all cell lines and primary tumors tested, whereas $M Y E O V$ expression was only detected in a subset of cell lines carrying MYEOV amplification. Additional experiments with the demethylating agent 5-aza-2'-deoxycytidine (5-AzaCd) suggest that $M Y E O V$ transcription is epigenetically silenced by methylation within the 11q13 amplicon.

\section{Materials and methods}

Cell lines and primary tumors of ESC

All 31 human ESC cell lines (KYSE series) were established from surgically resected tumors (Shimada et al. 1992), and maintained in RPMI-1640 supplemented with $10 \%$ fetal calf serum. Data from comparative genomic hybridization analyses involving 29 of these lines have been reported elsewhere (Pimkhaokham et al. 2000). After obtaining written consent from each patient as prescribed by the respective ethics committees, ESC tumor samples were collected from 48 independent patients treated at the University Hospitals of Kyoto and Keio in the formed style as approved.

Southern, dot, and Northern blot hybridizations

For Southern blot analysis, 10- $\mu \mathrm{g}$ aliquots of EcoRIdigested DNA extracted from each cell line or normal peripheral blood cells were electrophoresed in $0.8 \%$ agarose gels and transferred to nylon membranes (BIODYNE B, Nihon Pall, Tokyo, Japan). For dot blot analysis, 2- $\mu \mathrm{g}$ of DNA from each primary tumor, each cell line, or normal lymphocytes was denatured with $0.4 \mathrm{~N} \mathrm{NaOH}$, and then transferred to nylon membranes (BIODYNE B, Nihon Pall). For Northern blot analyses, $10-\mu \mathrm{g}$ samples of total RNA extracted from each cell line were size fractionated in $1.0 \%$ agarose $/ 0.67 \mathrm{M}$ formaldehyde gels and transferred to positively charged nylon membranes (Hybond-N+, Amersham Pharmacia Biotech, Tokyo, Japan).

Each membrane was hybridized with $\left[\alpha^{32} \mathrm{P}\right]-$ deoxycytidine triphosphate-labeled MYEOV cDNA under appropriate conditions, washed, and then exposed as described elsewhere (Janssen et al. 2000; Imoto et al. 2001a, 2001b). Subsequently, membranes were hybridized with cDNA probes for CCND1, EMS1, and the human glyceraldehyde-3-phosphate dehydrogenase gene $(G A P D H)$ as an internal standard. Autoradiographic signals were analyzed using a BAS-2500 image analyzer (Fuji film, Tokyo, Japan). After the background was subtracted, an intensity of each signal was evaluated with normalization divided by $G A P D H$ signal for each sample.

\section{5-AzaCd treatment}

To assess reactivation of MYEOV expression, we plated seven ESC cell lines (KYSE-30, 170, 200, 510, 790, 960, and 1170) in six-well tissue culture plates $24 \mathrm{~h}$ before treatment. Cells were treated with $10 \mu \mathrm{M}$ of 5 -AzaCd (Sigma Chemical, St. Louis, MO, USA) as described by Kusaba et al. (1999), and harvested after 7 days. Total RNA was extracted, electrophoresed, northern blotted, and analyzed as described earlier.

\section{Results}

Amplification of MYEOV, CCND1, and EMS1 in ESC cell lines and primary tumors

As shown in Fig. 1A, 20 of $31(64.5 \%)$ cell lines showed coamplification of MYEOV, CCND1, and EMS1. One cell line showed small amplification of EMS1 alone (KYSE-30), and the remaining 10 cell lines (KYSE-70, 150, 170, 190, $270,350,450,850,1190$, and 1440 ) showed no amplification of these genes. Because of a limited amount of DNA, we carried out dot blot analysis in primary ESC tumors, and identified that MYEOV and CCND1 are always coamplified in 6 of 48 tumors (12.5\%) (Fig. 2).

Differential expression pattern between $M Y E O V$ and $C C N D 1$ in ESC cell lines

Northern blot analysis revealed that all 20 cell lines with amplification of CCND1 showed its consequent overexpression. One cell line (KYSE-350) without CCND1 amplification also showed high expression of this gene. Under the same exposure conditions (24h), MYEOV expression was detected in the KYSE-1240 cell line only (data not shown). After longer exposure (14 days), we were able to detect marked MYEOV expression levels in nine cell lines (KYSE-170, 220, 410, 450, 1240, 1250, 1260, 2270, and 2650) and low to moderate $M Y E O V$ expression levels in 7 lines (KYSE-30, 150, 270, 350, 790, 850 and 2400) (Fig. 1B). Eleven lines (KYSE-110, 140, 180, 200, 510, 520, 590, 770, 890,960 , and 1170) exhibiting coamplification of $M Y E O V$ and CCND1 did not show any MYEOV expression, although all of them showed elevated or high levels of CCND1 expression (Fig. 1B). Seven cell lines showed $M Y E O V$ expression without concomitant $M Y E O V$ amplification (KYSE-30, 150, 170, 270, 350, 450, and 850). 


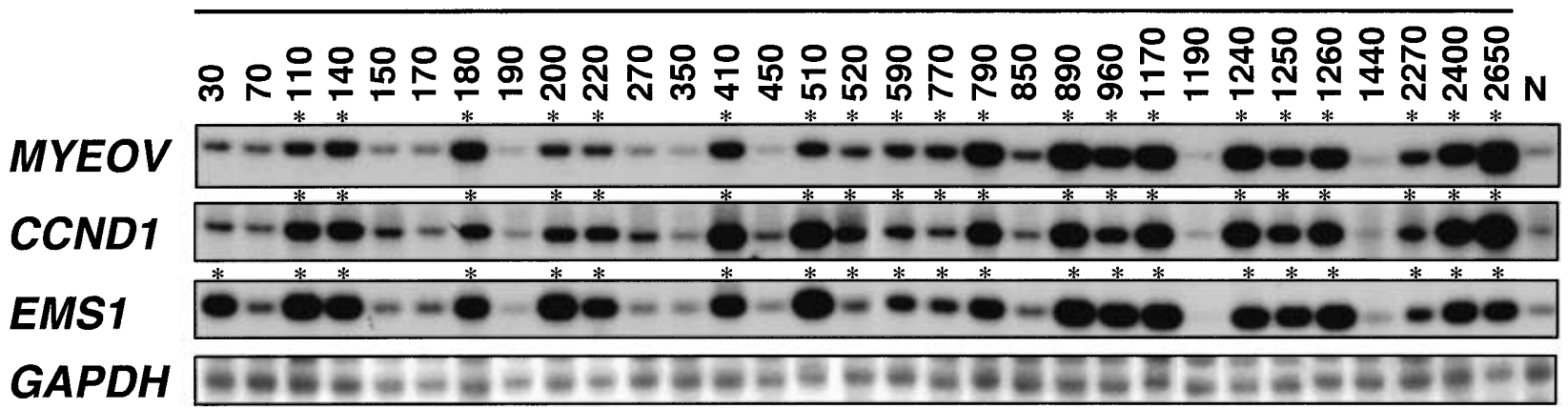

B

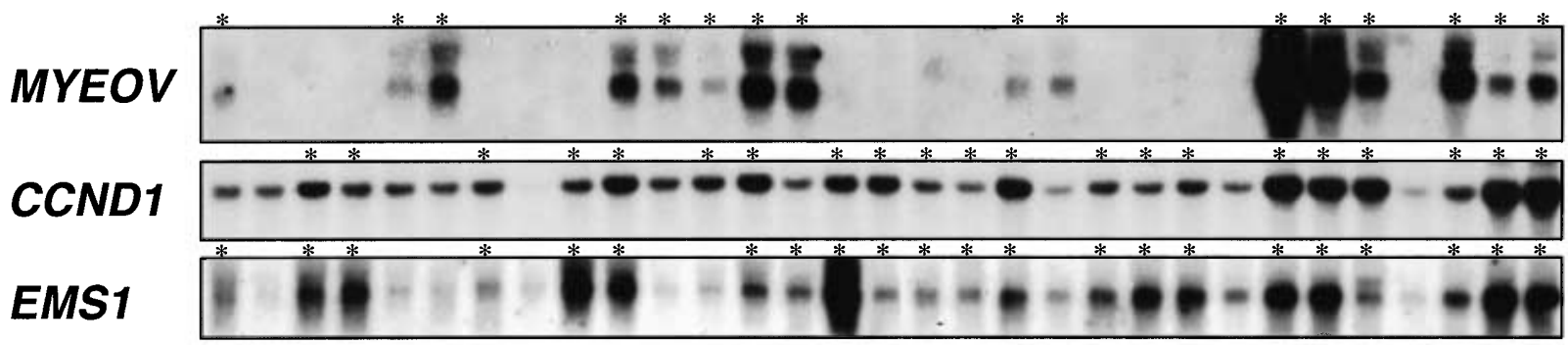

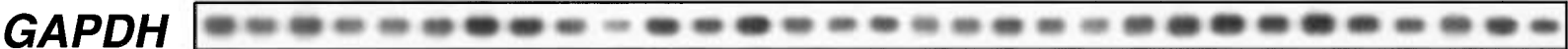

Fig. 1A,B. Southern and Northern blot analyses of $M Y E O V, C C N D 1$, and EMS1 in esophageal squamous cell carcinoma (ESC) cell lines. A Representative results of Southern blot analyses using $M Y E O V$, $C C N D 1$, and $E M S 1$ probes and an internal control $(G A P D H)$ probe in 31 ESC cell lines and normal peripheral lymphocytes from a healthy donor $(N)$. Asterisks indicate amplification. B Representative results of
Northern blot analyses using $M Y E O V, C C N D 1$, and $E M S 1$ probes and an internal control $(G A P D H)$ probe in the same panel of ESC cell lines. The exposure times for the MYEOV, CCND1, and EMS1 probes were 14 days, $24 \mathrm{~h}$, and $36 \mathrm{~h}$, respectively. Asterisks indicate overexpression. Note the close correlation between DNA copy-number status and the expression level of $C C N D 1$ and EMS1, but not MYEOV
Fig. 2. Representative results of dot blot analysis of $M Y E O V$ and $C C N D 1$ genes in primary ESC tumors. Cases 2 and 6 showed strong amplification signals with both MYEOV and CCND1 (arrowheads). GAPDH served as trol (peripheral blood leukocytes of a healthy donor) a control probe. $N$, Normal con-

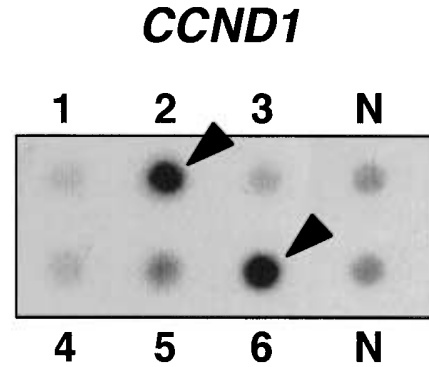

Similar to CCND1, high expression of EMS1 was consistently observed in cell lines with its amplification, although one cell line, KYSE-450, showed high expression of EMS1 in the absence of its amplification.

Restoration of $M Y E O V$ expression after treatment with 5-AzaCd

A comparison between our Southern and Northern blot data revealed some discrepancies; for example, in some cell lines $M Y E O V$ amplification occurred without concomitant $M Y E O V$ expression. These data suggested that $M Y E O V$ expression might be regulated epigenetically; for example, by methylation. Therefore, we examined restoration of
MYEOV
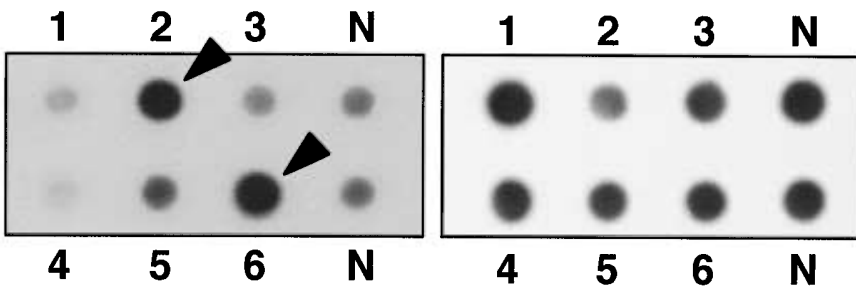

MYEOV expression in MYEOV amplification-positive/ expression-negative ESC cell lines after 5-AzaCd treatment. Seven different cell lines (KYSE-30, 170, 200, 510, 790, 960, and 1170), of which one line (KYSE170) showed high $M Y E O V$ expression without concomitant DNA amplification, were treated with 5-AzaCd. Despite 5-AzaCd treatment, $M Y E O V$ expression levels in the KYSE-170 cell line were barely changed. On the other hand, MYEOV expression was clearly restored in most of the other cell lines (Fig. 3). Restored MYEOV expression levels were very prominent in three cell lines (KYSE-510, 790, and 1170), all of which showed distinct amplification of $M Y E O V$, and their restored expression levels matched with their respective amplification levels (Fig. 3). 
KYSE cell lines

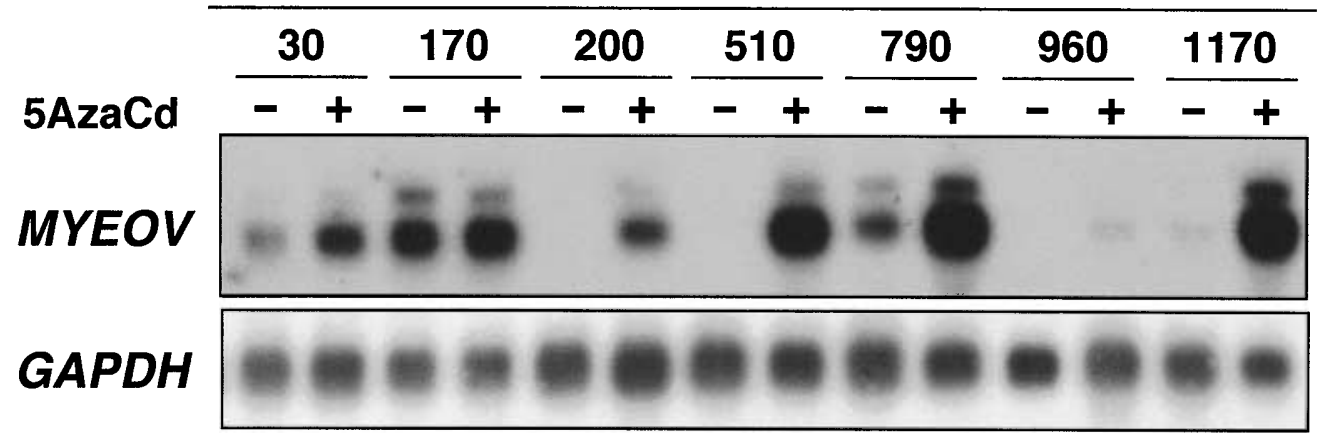

Fig. 3. Northern blot analysis of $M Y E O V$ in ESC cell lines treated with or without the DNA methylation inhibitor agent 5-aza-2'deoxycytidine (5-AzaCd). Cells were treated with $10 \mu \mathrm{M}$ of 5-AzaCd or vehicle for 7 days. GAPDH served as a control probe. Note the clear reconstitution of $M Y E O V$ expression after 5-AzaCd treatment in the ESC cell lines KYSE-510, 790, and 1170 at levels comparable to their

relative DNA amplification levels. In KYSE-960, the MYEOV expression level was partially restored, but at a level incomparable to its amplification status. In KYSE-170 exhibiting high $M Y E O V$ expression without its amplification, $M Y E O V$ expression levels were not altered after 5-AzaCd treatment

\section{Discussion}

Amplification of the chromosomal region of $11 \mathrm{q} 13$ is commonly observed in various human cancers, including breast, bladder, ESC, and head and neck tumors (Knuutila et al. 1998). The amplicon involved has been reported to harbor several genes, including CCND1, FGF4 (HSTF1), FGF3 (INT2), and EMS1. Among them, the role of CCND1 and EMS1 in the formation of the amplification has been well established (Schuuring 1995). CCND1 is located within $60 \mathrm{~kb}$ of $F G F 4$ (http://www.ncbi.nlm.nih.gov/cgi-bin/ Entretz/map-search), whereas EMS1 maps at least $800 \mathrm{~kb}$ telomeric to CCND1 (Hui et al. 1997). Amplification data for CCND1, FGF3, and EMS1 in a cohort of 747 breast cancers showed coamplification of $C C N D 1$ and $F G F 3$, but not of EMS1 and CCND1, or EMS1 and FGF3 (Hui et al. 1997). In addition, EMS1 amplification without concomitant CCND1 and/or FGF3 amplification was reported in a significant fraction (44\%). Independent amplification of CCND1 and EMS1 has been reported in other types of tumors as well (Schuuring 1995; Meredith et al. 1995; Rodrigo et al. 2000). Consistent with those data, our results show that EMS1 could also be found amplified solely, although it was coamplified with CCND1 in most cases, whereas $M Y E O V$ was always coamplified with $C C N D 1$ in cell lines and primary tumors of ESC. Taken together, our data are consistent with an amplification core spanning at least the region between $M Y E O V$ and FGF3 in ESC. Several genes activated by DNA amplification in the $11 \mathrm{q} 13$ region may be located within this core region.

DNA methylation is thought to play a key role in the regulation of gene expression, and various genes show an inverse relationship between DNA methylation and transcription in both normal and malignant cells (Boyes and Bird 1992; Ponder 2001; Baylin et al. 2001). Our results showed prominent restoration of $M Y E O V$ expression after treatment with 5-AzaCd in three cell lines (KYSE-510, 790, and 1170), all of which exhibited distinct amplification of this gene, supporting the hypothesis that, despite its amplification, aberrant methylation of the $M Y E O V \mathrm{CpG}$ islands inhibited its expression. Indeed, restored $M Y E O V$ expression levels were almost proportional to the level of $M Y E O V$ amplification in each of the cell lines after 5AzaCd treatment. Because the genomic structure of the $M Y E O V$ gene, including its promoter region, has not yet been characterized, we could not evaluate the methylation status of the promoter region regulating $M Y E O V$ expression. Therefore, the possibility remains that the restored MYEOV expression by 5 -AzaCd treatment may be induced indirectly via activating the expression of trans-acting factor(s) regulating $M Y E O V$ transcription. Another possible mechanism to modulate the transcription of the $M Y E O V$ gene independent of its genomic copy number would be by histone deacetylation (Struhl 1998). Histone deacetylases (HDACs) regulate the acetylation status of the core nucleosomal histones, which regulate the transcriptional activity of certain genes, and HDAC activity is generally associated with transcriptional repression (Struhl 1998). However, our preliminary study using Tricostatin A, a potent HDAC inhibitor, with or without 5-AzaCd, did not alter $M Y E O V$ expression, excluding this possibility (unpublished data). Further study will be necessary to determine the precise mechanisms for silencing $M Y E O V$ expression in tumor cells with its amplification.

Amplified chromosomal regions usually span a region of several megabases, consist of several amplification units, and harbor many different genes (Schuuring 1995; Schwab 1998). However, the number of target genes activated via amplification is usually limited (Imoto et al. 2001b; Schuuring et al. 1992; Yasui et al. 2001; Nakakuki et al. 2002; Platzer et al. 2002). Despite amplification, several genes in the amplicon are not upregulated, even though they are previously nominated candidate targets for amplification or known to be oncogenes (Platzer et al. 2002); the reasons have not yet been elucidated. Our results suggest that DNA methylation might be a mechanism for escaping upregulation of genes involved in amplification. Further 
studies will be necessary to clarify whether DNA methylation plays an important role in gene silencing of different genes, both within the same amplicon and among different amplicons on different chromosomes.

Acknowledgments This work was supported by Grants-in-Aid for Scientific Research on Priority Areas (C, to I.I. and J.I.) and for Basic Research (B2, to J.I.) from the Ministry of Education, Culture, Sports, Science, and Technology of Japan; by Grant-in-Aid from the Ministry of Health and Welfare of Japan (to J.I.); and by a grant of the Dr. Mildred Scheel Stiftung für Krebsforschung 10-1855-Ja2 (to J.W.G.J.).

\section{References}

Adelaide J, Monges G, Derderian C, Seitz JF, Birnbaum D (1995) Oesophageal cancer and amplification of the human cyclin $\mathrm{D}$ gene CCND1/PRAD1. Br J Cancer 71:64-68

Baylin SB, Esteller M, Rountree MR, Bachman KE, Schuebel K, Herman JG (2001) Aberrant patterns of DNA methylation, chromatin formation and gene expression in cancer. Hum Mol Genet 10: 687-692

Boyes J, Bird A (1992) Repression of genes by DNA methylation depends on $\mathrm{CpG}$ density and promoter strength: evidence for involvement of a methyl-CpG binding protein. EMBO J 11:327-333

Chesi M, Kuehl WM, Bergsagel PL (2000) Recurrent immunoglobulin gene translocations identify distinct molecular subtypes of myeloma. Ann Oncol 11(Suppl 1):131-135

Hui R, Campbell DH, Lee CS, McCaul K, Horsfall DJ, Musgrove EA, Daly RJ, Seshadri R, Sutherland RL (1997) EMS1 amplification can occur independently of CCND1 or INT-2 amplification at 11q13 and may identify different phenotypes in primary breast cancer. Oncogene 15:1617-1623

Imoto I, Pimkhaokham A, Fukuda Y, Yang ZQ, Shimada Y, Nomura N, Hirai H, Imamura M, Inazawa J (2001a) Sno is a probable target for gene amplification at 3q26 in squamous-cell carcinomas of the esophagus. Biochem Biophys Res Commun 286:559-565

Imoto I, Yang ZQ, Pimkhaokham A, Tsuda H, Shimada Y, Imamura M, Ohki M, Inazawa J (2001b) Identification of cIAP1 as a candidate target gene within an amplicon at 11q22 in esophageal squamous cell carcinomas. Cancer Res 61:6629-6634

Janssen JW, Braunger J, Ballas K, Faust M, Siebers U, Steenvoorden AC, Bartram CR (1999) Spectrum of transforming sequences detected by tumorigenicity assay in a large series of human neoplasms. Int J Cancer 80:857-862

Janssen JW, Vaandrager JW, Heuser T, Jauch A, Kluin PM, Geelen E, Bergsagel PL, Kuehl WM, Drexler HG, Otsuki T, Bartram CR, Schuuring E (2000) Concurrent activation of a novel putative transforming gene, myeov, and cyclin D1 in a subset of multiple myeloma cell lines with $\mathrm{t}(11 ; 14)(\mathrm{q} 13 ; \mathrm{q} 32)$. Blood 95:2691-2698

Knuutila S, Bjorkqvist AM, Autio K, Tarkkanen M, Wolf M, Monni O, Szymanska J, Larramendy ML, Tapper J, Pere H, El-Rifai W, Hemmer S, Wasenius VM, Vidgren V, Zhu Y (1998) DNA copy number amplifications in human neoplasms: review of comparative genomic hybridization studies. Am J Pathol 152:1107-1123
Kusaba H, Nakayama M, Harada T, Nomoto M, Kohno K, Kuwano M, Wada M (1999) Association of $59 \mathrm{CpG}$ demethylation and altered chromatin structure in the promoter region with transcriptional activation of the multidrug resistance 1 gene in human cancer cells. Eur J Biochem 262:924-932

Meredith SD, Levine PA, Burns JA, Gaffey MJ, Boyd JC, Weiss LM, Erickson NL, Williams ME (1995) Chromosome 11q13 amplification in head and neck squamous cell carcinoma. Association with poor prognosis. Arch Otolaryngol Head Neck Surg 121:790-794

Mitelman F, Mertens F, Johansson B (1997) A breakpoint map of recurrent chromosomal rearrangements in human neoplasia. Nat Genet 15:417-474

Nakakuki K, Imoto I, Pimkhaokham A, Fukuda Y, Shimada Y, Imamura M, Amagasa T, Inazawa J (2002) Novel targets for the 18 p11.3 amplification frequently observed in esophageal squamous cell carcinomas. Carcinogenesis 23:19-24

Nakamura S, Yatabe Y, Seto M (1997) Cyclin D1 overexpression in malignant lymphomas. Pathol Int 47:421-429

Pimkhaokham A, Shimada Y, Fukuda Y, Kurihara N, Imoto I, Yang ZQ, Imamura M, Nakamura Y, Amagasa T, Inazawa J (2000) Nonrandom chromosomal imbalances in esophageal squamous cell carcinoma cell lines: possible involvement of the ATF3 and CENPF genes in the 1q32 amplicon. Jpn J Cancer Res 91:1126-1133

Platzer P, Upender MB, Wilson K, Willis J, Lutterbaugh J, Nosrati J, Willson JKV, Mack D, Ried D, Markowitz S (2002) Silence of chromosomal amplifications in colon cancer. Cancer Res 62:1134-1138

Ponder BA (2001) Cancer genetics. Nature 411:336-341

Rodrigo JP, Garcia LA, Ramos S, Lazo PS, Suarez C (2000) EMS1 gene amplification correlates with poor prognosis in squamous cell carcinomas of the head and neck. Clin Cancer Res 6:3177-3182

Ronchetti D, Finelli P, Richelda R, Baldini L, Rocchi M, Viggiano L, Cuneo A, Bogni S, Fabris S, Lombardi L, Maiolo AT, Neri A (1999) Molecular analysis of 11q13 breakpoints in multiple myeloma. Blood 93:1330-1337

Schuuring E (1995) The involvement of the chromosome 11q13 region in human malignancies: cyclin D1 and EMS1 are two new candidate oncogenes - a review. Gene 159:83-96

Schuuring E, Verhoeven E, Mooi WJ, Michalides RJ (1992) Identification and cloning of two overexpressed genes, U21B31/ $P R A D 1$ and $E M S 1$, within the amplified chromosome 11q13 region in human carcinomas. Oncogene 7:355-361

Schwab M (1998) Amplification of oncogenes in human cancer cells. Bioessays 20:473-479

Shimada Y, Imamura M, Wagata T, Yamaguchi N, Tobe T (1992) Characterization of 21 newly established esophageal cancer cell lines. Cancer 69:277-284

Shinozaki H, Ozawa S, Ando N, Tsuruta H, Terada M, Ueda M, Kitajima M (1996) Cyclin D1 amplification as a new predictive classification for squamous cell carcinoma of the esophagus, adding gene information. Clin Cancer Res 2:1155-1161

Struhl K (1998) Histone acetylation and transcriptional regulatory mechanisms. Genes Dev 12:599-606

Yasui K, Imoto I, Fukuda Y, Pimkhaokham A, Yang Z-Q, Naruto T, Shimada Y, Nakamura Y, and Inazawa J (2001) Identification of target genes within an amplicon at 14q12-q13 in esophageal squamous cell carcinoma. Genes Chromosomes Cancer 32:112-118

Yoshida T, Sakamoto H, Terada M (1993) Amplified genes in cancer in upper digestive tract. Semin Cancer Biol 4:33-40 